\title{
Dental Medical Waste, Promising Source of Adult Stem Cells for Tissue Regeneration
}

\author{
Maryam Rezai Rad, DDS, PhD \\ Dental Research Center, Research Institute of Dental Sciences, School of Dentistry, \\ Shahid Beheshti University of Medical Sciences, Tehran, Iran
}

Utilization of patient-specific stem cells provides a novel treatment approach in regenerative medicine. Although embryonic stem cells (ESCs) are pluripotent and can differentiate into any cells found within the body, use of ESCs raises various issues including safety and ethical concerns. Induced pluripotent stem cells (iPSCs) have also been studied and reported. Experimental evidence indicates that similar to ESCs, iPSCs cannot be used safely for human therapies. Tumorigenicity after transplantation of ESCs and iPSCs is the major threat in human therapies. Such tumorigenicity is likely due to their pluripotency. Currently, it is not clear whether the obstacles to use of ESCs and iPSCs for human treatment can be overcome. There is still a long way to go to overcome such obstacles. Stem cells, however, can be isolated from adult tissues as well. Due to lack of tumorigenicity after transplantation, they are considered safe for human therapies. Tissue-derived adult stem cells (ASCs) are multipotent and can differentiate into limited types of cells, usually within the same germ layer of tissues in which they reside. Therefore, isolation of ASCs from various tissues is necessary for regeneration of different tissues. In this regard, ASCs derived from numerous tissues have been studied.

The most studied adult stem cells are mesenchymal stem cells, derived from bone marrow aspirate (BMMSCs). This stem cell population providesa valuable autogenous cell source for cell-based tissue engineering. However, the search for an alternative source is necessary, since obtaining BMMSCs requires an invasive procedure of bone marrow aspiration. One of the promising alternative solutions would be the isolation of adult stem cells from tissues discarded during the course of medical practice.

Recently, considerable attention has been paid to stem cells from dental tissues which can be obtained from discarded medical wastes during dental practices. Most people have an impacted third molar that needs to be extracted to avoid inflammation or orthodontics treatments. Such extracted teeth can be used for isolation of dental stem cells (DSCs). Moreover, deciduous teeth, another readily available dental waste, have been shown to be an incredible potential source of adult stem cells.

The majority of previous studies have demonstrated that DSCs have potential applications in various dental treatments (e.g., periodontal and maxillofacial regeneration, osseous integration of implants, and dental pulp regeneration). However, recent studies have suggested that such stem cells also have much potential for diverse non-dental medical applications.

Despite introducing DSCs as aneasily accessible autogenous source of adult stem cells for tissue regenerative medicine, stem cell technology is still in its infancy. Hence, establishing the dental bank for preservation of these adult stem cells, tissue, or teeth will provide significant potential for patients to benefit from the advanced therapies of tomorrow. 